\title{
TURBULENCE AND MAGNETIC FIELDS IN MOLECULAR CLOUDS
}

\author{
R.N. HENRIKSEN \\ service d'astrophysique, batiment 528 \\ c.e.n. saclay, 91191 gif sur yvette \\ cedex, france \\ E-mail @32779 :: henriksen.
}

Keywords : Gravitation, Turbulence, Magnetic, Molecular, Clouds

Abstract. In this paper I first review some of the simple structural concepts associated with compressible turbulence. In particular the hierarchical or self-similar fractal structure to be expected is formulated in a manner readily compared to the observations, and to previous work. In the next section I present the first results of a wavelet analysis on molecular clouds, which seem to comfirm the hierarchical scaling. I conclude with an extention of the theory to include magnetic fields. This latter theory represents an alternative to the more conventional dynamo theory.

\section{Compressible Turbulence and Fractal Hierarchies}

All quantitative science depends on a quantitative description of the phenomenon in question. In the case of the interstellar molecular clouds various authors (Zinnecker,1984; Henriksen and Turner, 1984; Henriksen, 1986 ; Elmegreen, 1989) have suggested that the phenomenon is sufficiently complex and hierarchical to be termed 'turbulent'. In such a framework the concept of fractal geometry is known to permit a precise and quantitative description, although in general an infinite set of parameters is required. Fortunately these can be greatly restricted by selection on the basis of their physical significance. I shall begin by introducing the parameters that I have tried to use consistently in my own descriptions of the molecular cloud turbulence, taking care to emphasize their physical significance.

The fractal capacity or Hausdorf dimension $D$ is defined, in a discrete model, in terms of the number of objects of scale $\ell$ in the structure say $n(\ell)$. Thus if two scales $\ell$ and $L$ have the ratio $r=\ell / L$, then the number of fragments of size $\ell$ per fragment of size $L$ is given by

$$
n(\ell) / n(L)=r^{-D},
$$

where for a self-similar structure $r$ is constant over all the various stages. Notice that the pieces may be irregular in detailed shape, but that in a statistical average they should be characterizable by one scale in this simple picture. Otherwise anisotropic fractal structure is required.

It is important to note that on passing to a continuum model of the structure wherein $N(L)$ gives the total number of fragments having $\ell \leq L$, the correspondence with the discrete $n(\ell)$ is the logarithmic derivative of $N$ with respect to $\ell$. For we clearly must associate equal ratios with equal differences in the logarithmn. Thus

$$
\frac{d N}{d \ln (\ell)} \propto \ell^{-D}
$$

for the continuum model. 
Now let us in addition introduce a dimension(Henriksen, 1986) that gives the variation of the mean density on a scale $\ell$ with $\ell$. By mean density we shall mean here $\bar{\rho}(\ell)$ such that the mass of an element of scale $\ell$ is

$$
M(\ell)=\bar{\rho}(\ell) \ell^{3} .
$$

Previously, I have used the density of a subscale fragment (Henriksen 1986) so that 3 in equation (3) is replaced by a fractal dimension. I hereby renounce that somewhat confusing procedure! Then I introduce $D_{\rho}$ so that

$$
\bar{\rho}(\ell) \propto \ell^{-D_{\rho}} .
$$

These two dimensional indices allow us to construct the dependence of various significant physical quantities on scale. Thus the total mass on a scale $\ell, n(\ell) M(\ell)$ is related to that on a superscale $L$ as

$$
n(\ell) M(\ell)=n(L) M(L) r^{\left(3-\left(D+D_{\rho}\right)\right)} .
$$

It is convenient to introduce an 'aggregation index'

$$
i_{1} \equiv 3-\left(D+D_{\rho}\right),
$$

such that $i_{1}=0$ in a pure aggregation or fragmentation hierarchy where each superscale is composed solely of fragments of subscale $\ell$. In this case $D$ and $D_{\rho}$ are not independent. However such a case is rather idealised for the molecular clouds where one expects there to be lower density material filling the voids between the subscale density peaks, that is 'fragments'. Such a 'smoothed' aggregation hierarchy requires the two independent dimensions, and the difference between $i_{1}$ and 0 is a measure of the deviation from a pure geometric hierarchy, with $i_{1}>0$ implying that there is mass between the subscale fragments (in the uniform limit $i_{1}=3$ ).

Moreover, following Henriksen and Turner (1984), the collision time between two fragments of scale $\ell$ moving in a volume $L^{3}$ is a measure of the dynamical coupling between the scales when compared to the dynamical time on the scale $L$. An interesting example is afforded by a simple binary hierarchy, wherein a resonance between the period of one binary and the time scale of the encounter between two such binaries on the next superscale can be expected to produce the most pronounced exchange of angular momentum and energy between the scales (see e.g. Lattanzio and Henriksen,1988). We calculate this quantity in a straightforward way as $\left(v_{L}\right.$ is the velocity dispersion on the scale $\left.L\right)$

$$
t_{c}(\ell) \equiv \frac{1}{\left(\frac{n(\ell)}{n(L) L^{3}} \times \ell^{2} v_{L}\right)},
$$

or on using equation (5) and the definitions above and after multiplying this expression top and bottom by $M(\ell)$, one obtains

$$
t_{c}(\ell)=\frac{L}{v_{L}} \times r^{(D-2)}
$$


In this way one defines a 'dynamical coupling index' $i_{2}$ such that

$$
i_{2} \equiv D-2 \text {. }
$$

When $i_{2}=0$, we see that there is strong dynamical coupling wherein $t_{c}=L / v_{L}$. When $i_{2}<0$, the subscale collision time is longer than the superscale dynamical time, which reduces the resonance between the scales, while when $i_{2}>0$ the collision time is relatively short and one expects collisional damping to terminate the dynamical cascade ultimately. In Henriksen and Turner (1984) $i_{1}=0$ so that a pure aggregation hierarchy was assumed implicitly, and thus $i_{2}=1-D_{\rho}$. Subsequently the imposition of the dynamical condition $t_{c}=L / v_{L}$, so that $i_{2}=0$ allowed HT to deduce that $D_{\rho}=1$ and that the velocity scaling was $\propto \ell^{1 / 2}$. They did not use the index $D$, but in Henriksen (1986) we now see that $D$ is constrained to be 2 if the pure aggregation hierarchy is maintained. For general $D$ and $D_{\rho}$, neither one of the indices $i_{1}$ and $i_{2}$ are 0 , and the scaling in the turbulence depends only on the constants $\lambda \equiv \bar{\rho} \ell^{D_{\rho}}$ and Newton's constant $G$. This general predicted scaling (Henriksen, 1990) is

$$
\begin{aligned}
v(\ell) & =\sqrt{G \lambda} \ell^{\left(1-D_{\rho} / 2\right)} u(\xi), \\
\rho(\ell) & =\lambda \ell^{-D_{\rho}} \mu(\ell), \\
B & =\sqrt{G \lambda^{2}} \ell^{\left(1-D_{\rho}\right)} b(\xi), \\
p & =G \lambda^{2} \ell^{2\left(1-D_{\rho}\right)} P(\xi), \\
\xi & =\frac{G \lambda t^{2}}{\ell^{D_{\rho}}} .
\end{aligned}
$$

The indices $i_{1}$ and $i_{2}$ remain useful as indicators of the respective physical hierarchies discussed above.

One of the interesting applications of these ideas is the predicted relation ( Henriksen, 1986) between these indices and the index of the scaling portion of the IMF defined as

$$
\alpha_{*} \equiv-\log \left(d N / d \log M_{*}\right) \text {. }
$$

As in Henriksen (1986) but using now $M(\ell) \propto \ell^{3-D_{\rho}}$, one obtains directly from equation (2) that for the clouds

$$
\alpha=D /\left(3-D_{\rho}\right) \text {. }
$$

Subsequently Henriksen(1986) assumed that the mass of a star formed in a cloud would be proportional to the mass of the cloud. However at this meeting Larson has argued that the relation may be closer to

$$
M_{*} \propto M^{0.43} \text {. }
$$

In general we may want to introduce a degree of freedom here (see e.g. Zinnecker 1989) and call this power $f$. Then we have simply that

$$
\alpha_{*}=\alpha / f=D /\left(f\left(3-D_{\rho}\right)\right)
$$


If $\alpha=0.6$ as suggested at this conference by Elisabeth Lada, then $\alpha_{*}=1.4$ with $f=0.43$. In such a case $D=1.2$ from (9) if $D_{\rho}=1$. This does not however agree with more direct measures of the dimension $D$ (see the next section). At the other extreme, $f=1, D_{\rho}=1$, and $D=2.7$ give $\alpha=1.35$, the Salpetre value, which dimension was the suggestion of Henriksen(1986). This value however appears to overestimate the importance of the clouds of small mass relative to the value found by Lada and others.

\section{Wavelet Analysis}

In this section I wish to present what I believe is the first application of wavelet transform theory to a description of molecular clouds. The full description of this work is to be found in a paper by Gill and Henriksen (1990). Our intention is to use the velocities characterising emissivity peaks as the third coordinate for these peaks orthogonal to the plane of the sky. In this $\Delta V \cdot-\ell-b$ 'phase' space the wavelet analysis allows us to measure a dimension directly. Even without further interpretation of the velocity-scale relation, a fractional but well defined value provides evidence for a scaling fractal hierarchy in phase space indicative of turbulent processes.

I proceed by presenting briefly the wavelet analysis in its simplest form. A more general discussion can be found for example in Argoul et al. (1989). One works either in the space of 'scales' $a$ (1/a is the magnification) or in position space $\vec{b}$ corresponding to wave vector space and position space of ordinary Fourier analysis. Here we have supposed that the scaling is isotropic so that it is characterised by a single parameter, and morover we do not rotate our spatial axes from point to point, consistent with this presumed isotropy. One would not expect to obtain a convergent result if either of these constraints are badly broken. We have in mind the representation of real functions over the plane of the sky in terms of carefully chosen base functions (the analysing wavelets) $g(\vec{y})$. The wavelet that we consider best for our purposes to date is the so-called Mexican Hat wavelet which has the form

$$
g_{M}(\vec{y})=\left(2-\frac{\vec{y}^{2}}{a^{2}}\right) e^{-\left(\frac{\vec{t}^{2}}{2 a^{2}}\right)} .
$$

In order that the transform theorem hold, the wavelet must satisfy in practice

$$
\int g(\vec{y}) d \vec{y}=0
$$

which is the case for the Mexican Hat. In addition it is worth noting that $g_{M}=0$ where $\vec{y}=\sqrt{2} a$, and that it is 2 at the origin but $-2 / e^{2}$ at the position of the negative maximum $2 a$. The simplest statement of the wavelet transform theorem now reads for a properly behaved function $\rho(\vec{x})$;

$$
\begin{aligned}
T_{g}(a, \vec{b}) & =\int g\left(\frac{\vec{x}-\vec{b}}{a}\right) \rho(\vec{x}) d \vec{x}, \\
\rho(\vec{x}) & =K \int T_{g}(a, \vec{x}) a^{-3 / 2} d a .
\end{aligned}
$$


Here $K$ is a numerical constant which need only be calculated once.

For our purposes we work with the wavelet transform $T_{g}(a, \vec{b})$ itself since it possesses the important property of scaling with the analysed function. That is if $\rho(\lambda \vec{x}) \propto \lambda^{\alpha} \rho(\vec{x})$ then

$$
T_{g}(\lambda a, \vec{b}) \propto \lambda^{\alpha} T_{g}(a, \vec{b}) .
$$

Thus a plot of $\log T_{g}$ versus $\log a$ will yield a straight line of slope $\alpha$ if indeed the function being analysed scales in the assumed manner. Such a demonstration of scaling is important in itself, although the physical interpretation is more problematical and clearly dependent on the function being analysed.

However, a second remarkable property of the wavelet transform is that some positional information is retained even at a single value of the scale $a$. In fact in Gill and Henriksen (1990) it is shown that if the maximum scale $a_{d}$ to which the well defined straight line in the $\log T_{g}-\log a$ extends is used, then $T_{g}\left(a_{d}, \vec{b}\right)$ maps the dominant structure of the analysed function over the plane of the sky reasonably well.

We have applied this technique to ${ }^{13} \mathrm{CO}$ spectra of the $L 1551$ star forming region kindly loaned to us for this purpose by Moriarty-Schieven and Snell (1988). Approximately one half of the some 1500 spectra had sufficiently good $\mathrm{S} / \mathrm{N}$ ratios that the velocity at the emissivity peak could be identified both by eye and by fitting a Gaussian. There were essentially no incidences of well defined multiple peaks in the data, so that on average one emissivity peak was observed per line of sight.

The spectra were distributed over a $42 \times 44$ step grid which included the region of ${ }^{12} \mathrm{CO}$ molecular outflow and the source IRS 5 itself. The high velocity cloud that overlaps the region was removed from the velocity data as in Moriarty-Schieven and Snell (1988), and the mean velocity of the remaining $L 1551$ cloud was also subtracted from the peak velocity data. The resulting set of corrected peak velocities constituted the function $\rho(\vec{x})$ that was analysed using equations (10) and (11). We used an average over all points $\vec{b}$ on the grid for each value of $a$ (see figure caption) so that we postulate a homogeneous scaling on the grid (or subgrid). The calculation was actually done using a Fast Fourier Transform over $\vec{b}$ for each scale $a$, making use of the fact that equation (11) is recognisably a convolution integral. Finally the transform was inverted and the result is shown in figure 1.

One sees that these emissivity peak velocities do show a scaling law indicative of hierarchical structure. If moreover we adopt the view that the peak velocity is a measure of the position of the region of dominant emission orthogonal to the plane of the sky (see Gill and Henriksen,1990 for a justification), then the transform makes a statistical estimate of the rate at which the number of such emissivity peaks in a volume increases with the characteristic scale $a$. That is, we obtain a direct estimate of the fractal capacity or Hausdorf dimension. This gives $D=2.42 \pm 0.01$ in the notation used above in section 1 if we adopt the value found for the region outside the molecular outflow only, and $D=2.35 \pm 0.01$ for the average over the entire cloud.

On referring back to our discussion of section 1 , we see that this value of $D$ gives (equation 9 ) an $\alpha$ of either 1.21 or 1.17 respectively, if $D_{\rho}=1$, which is roughly twice the value reported at this conference and previously from direct cloud counts. Moreover if indeed $f$ is significantly different from 1 , then $\alpha_{*}$ becomes ultimately too large (a value as 


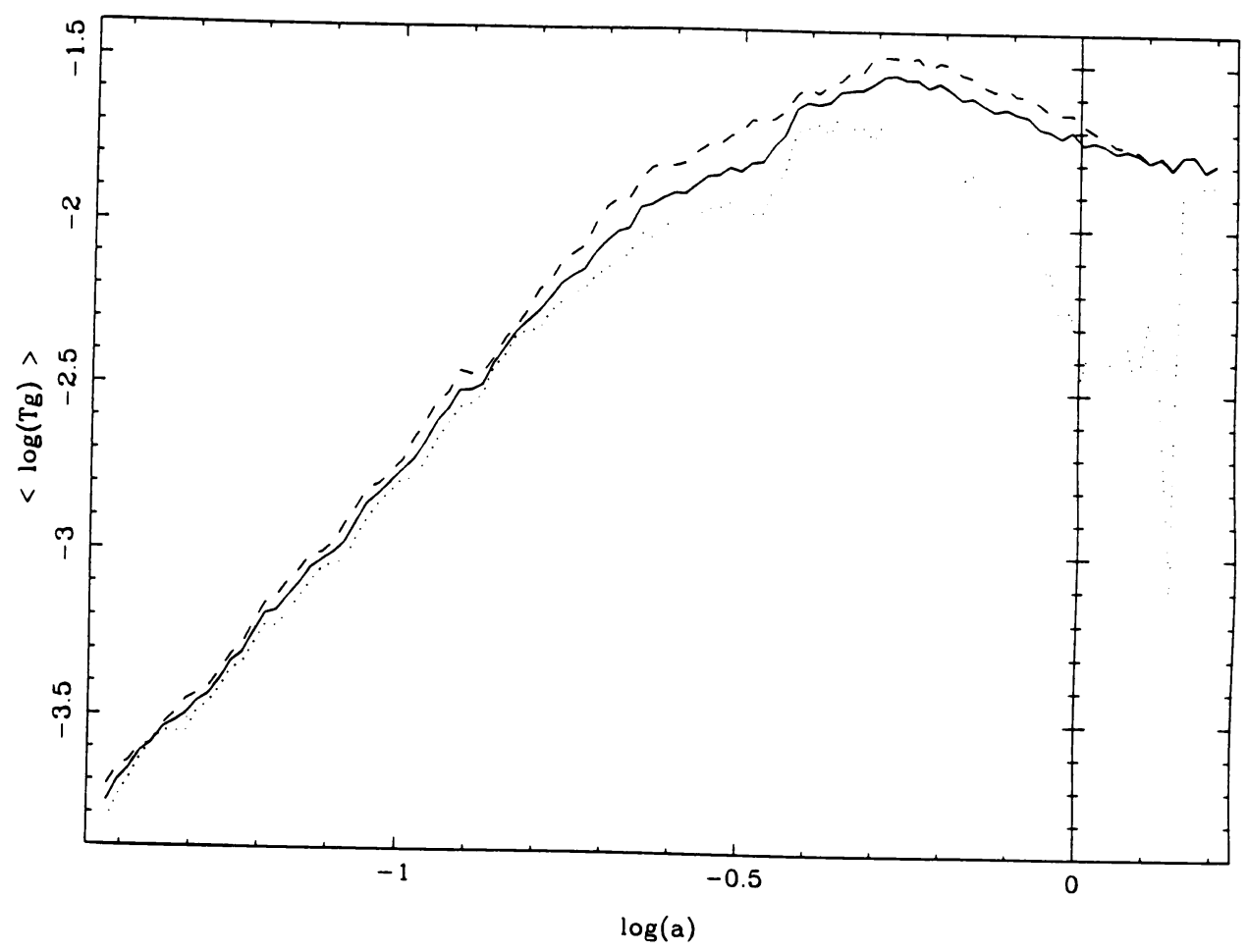

Fig. 1. The Mexican Hat wavelet transform of the peak velocity fluctuations of the ${ }^{13} \mathrm{CO}$ spectra in the $L 1551$ region. Positive values of $T_{g}$ are logarithmically averaged with a $S / N$ weighting scheme. The solid line is averaged over the entire cloud, the dashed line is averaged over the region outside the ${ }^{12} \mathrm{CO}$ molecular outflow while the dotted line is averaged over the outflow region alone. The scale parameter $\mathrm{a}$ is defined such that $\log (a)=-0.6 \leftrightarrow 0.5 p c$ in $L 1551$. The slopes for $\log (a)<-0.6=\log \left(a_{d}\right)$ are respectively, $2.35 \pm 0.01,2.42 \pm 0.01$, and $2.29 \pm 0.01$.

small as .7 is however acceptable). Thus this method of determining the index $D$ would be highly suspect were it not for the measurement of $D_{p}=1.36 \pm 0.02$ reported here by Falgarone as the fractal dimension in the plane of the sky. If the ${ }^{12} \mathrm{CO}$ measurements are optically thick, so that the dimension obtained is essentially that of a planar intersection with the true spatial distribution, then indeed $D_{p}=D-1$ and the agreement between these two highly independent measurements is essentially perfect. But to obtain this agreement, one must conclude that indeed $\Delta V \propto a$ in the region. This latter 'Hubble' law is an indication of turbulence, but is not the form usually quoted for molecular clouds $\left(\Delta V \propto a^{1 / 2}\right)$. Perhaps the velocity field throughout the region has been disturbed by the molecular outflow. In this connection I note that Prasad and Sreenivasan ( 1990) give $D=2.36 \pm 0.05$ for the Kolmogorov range of the turbulence associated with a water jet 
in quiet surroundings.

\section{Magnetic Turbulence}

If the indications of dynamical turbulence discussed in the preceeding sections are taken seriously, then a corresponding treatment of the magnetic field is necessary. In this section we wish to generalize the equations of Chaboyer and Henriksen (1990) for stochastic magnetic fields to include a Hubble type cloud divergence or contraction, as well as a feedback loop to the dynamical equations. This latter modification opens the question of true 'stochastic dynamos', although I do not pretend to close it here! The fundamental physical picture is that although the magnetic field may be ordered on the large scales, and again on the small protostellar scales, it is likely to be turbulent due to many competing dynamical processes on intermediate scales. On these scales then, the best global predictions for the magnetic field are probably in terms on the ensemble averaged two-point correlation functions in the spirit of Hoyng (1987a,b; 1988) and of Chandrasekhar (1955a,b; 1957). Observational evidence bearing on such a stochastic hierarchical image of magnetic stuctures is discussed in Chaboyer and Henriksen (1990), and recent results were presented by Heiles at this conference.

The fundamental approach to the description of the turbulence follows the self-similar model introduced by Henriksen and Turner (1984) following earlier work by Sedov (1982) and developed further in Henriksen (1986) and Henriksen (1988). The basic equations are the MHD equation with a non-constant turbulent resistivity $\eta$ as;

$$
\partial_{t} B^{i}=B^{k} \nabla_{k} v^{i}-\nabla_{k}\left(v^{k} B^{i}\right)-\nabla_{k}\left(\frac{\eta}{4 \pi} \nabla^{i} B^{k}\right)+\nabla_{k}\left(\frac{\eta}{4 \pi} \nabla^{k} B^{i}\right),
$$

where the velocity should be taken as

$$
v^{i}=u^{i}+H x^{i},
$$

and $H \equiv \dot{a} / a$ in terms of the overall scale factor $a(t)$. The coordinates $x^{i}$ are Eulerian. In the same notation the fundamental dynamical equation is

$$
\begin{aligned}
\partial_{t} u^{i} & =-\frac{1}{\rho} \nabla^{i} P-u^{k} \nabla_{k} u^{i}+F^{i}-H u^{i}-H\left(x^{k} \partial_{k}\right) u^{i} \\
& +\nu \nabla_{k} \sigma^{i k}+\zeta \nabla_{k}\left(g^{i k} \nabla_{a} u^{a}\right)+\sigma^{i k} \frac{\nabla_{k}(\rho \nu)}{\rho}+g^{i k}\left(\nabla_{a} u^{a}\right) \frac{\nabla_{k}(\rho \zeta)}{\rho},
\end{aligned}
$$

where

$$
\begin{aligned}
\sigma^{i k} & \equiv \nabla^{i} u^{k}+\nabla^{k} u^{i}-(2 / 3)\left(\nabla_{a} u^{a}\right) g^{i k}, \\
P & \equiv p+\frac{B^{2}}{8 \pi} \\
F^{i} & \equiv f^{i}+\frac{B^{k} \nabla_{k} B^{i}}{4 \pi \rho}, \\
f^{i} & \equiv-\nabla^{i} \Phi \\
\Phi & \equiv \phi-\frac{\ddot{a}}{2 a}(\vec{R})^{2} .
\end{aligned}
$$


Finally the continuity equation takes the form

$$
\partial_{t} \rho+\vec{\nabla} \cdot(\rho \vec{u})=-\vec{\nabla} \cdot(H \rho \vec{R}) .
$$

The analysis proceeds in terms of ensemble averaged second and third moments of these equations (see e.g. Henriksen 1988) expressed at two different points, $O$ and $O^{\prime}$. The results are written in terms of the two-point tensors

$$
\begin{aligned}
U^{i, j} & \equiv\left\langle B^{i} B^{\prime j}\right\rangle, \\
F^{i k, j} & \equiv\left\langle\frac{B^{i} B^{k} \rho^{\prime} u^{j}}{4 \pi \rho \rho^{\prime}}\right\rangle, \\
S^{i k, j} & \equiv\left\langle u^{i} B^{k} B^{\prime j}\right\rangle, \\
R^{i, j} & \equiv\left\langle u^{i} u^{\prime j}\right\rangle, \\
R^{i j, k} & \equiv\left\langle u^{i} u^{j} u^{\prime k}\right\rangle,
\end{aligned}
$$

together with their various contractions. One has also to use the properties of homogeneous and isotropic tensors (Henriksen,1988; Chaboyer and Henriksen,1990) which we shall not repeat here in the interests of brevity. When specific components of a tensor are given they refer to a right-handed reference system with the 1 axis along the line $O O^{\prime}$, and the $2-3$ axes in the orthogonal plane. An asterisk subscript emphasizes this frame specificity. One has no need to specify the absolute orientation of this system because of the assumed isotropy. The fundamental equations that we have derived for the stochastic dynamo are now;

$$
\begin{aligned}
\partial_{t} \tilde{\tilde{U}} & =\frac{\eta}{2 \pi} \nabla_{k} \nabla^{k} \tilde{\tilde{U}}+\frac{4}{L^{2}} \partial_{L}\left(L^{2} \tilde{\tilde{S}}\right)+H L \partial_{L} \tilde{\tilde{U}}, \\
\partial_{t} \tilde{R} & =2 \nabla_{k}\left(\delta_{i, j} \tilde{F}^{i k, j}\right)-2 \nabla_{k}\left(\delta_{i j} \tilde{R}^{i k, j}\right)+2 \nu \nabla_{k} \nabla^{k} \tilde{R}+2 \nu \nabla_{k}\left\langle\ln \rho \rho^{\prime}\right\rangle \nabla^{k} \tilde{R} \\
& +2 \tilde{F}_{i}^{i k,} \nabla_{k}\left\langle\ln \rho \rho^{\prime}\right\rangle+H L \partial_{L} \tilde{R}, \\
\left\langle\ln \rho \rho^{\prime}\right\rangle & =-6 a-2 D_{\rho} \ln L .
\end{aligned}
$$

In these equations $L$ stands for the distance between the pairs of points considered (i.e. the spatial lag), the notation () indicates $a^{2} \times()$, the index $D_{\rho}$ is defined in a manner closely analagous to that of the preceeding sections so that $\rho(L) \equiv \exp \left\langle\ln \rho \rho^{\prime}\right\rangle / 2 \propto L^{-D_{\rho}}$, and $S$ is a source term depending on the lack of mirror symmetry as

$$
S \equiv S_{*}^{12,2}-S_{*}^{21,2} \text {. }
$$

Now we recall that the assumption of isotropy implies that the traces of the tensors $R^{i, j}$, and $U^{i, j}$ suffice to define these tensors entirely (Henriksen,1988). Thus our equations would be complete were it not for the presence of the third order tensors, which is the familiar closure problem common to all such moment equations. The tensor $R^{i j, k}$ introduces one additional unknown $R_{*}^{11,1}$ say, as $F^{i j, k}$ introduces $F_{*}^{11,1}$. In addition one has both $S$ and $S_{*}^{1,11}$, although the latter does not yet appear explicitly. This means only that we have not yet specified the 'source' tensor completely. 
The process of self-similar closure advocated in Henriksen(1988) is capable of relating $S$ and $U$ as well as $R, R_{*}^{11,1}$ and $F_{*}^{11,1}$. However one additional hypothesis is required in general. As an example, one posible choice would be to write the symmetric and antisymmetric parts of $S^{i k, j}$ on $i k$ as

$$
S^{i k, j}=\text { constant } \times \rho(L) F^{i k, j}+S\left(\frac{\delta^{k j} L^{i}}{L}-\frac{\delta^{i j} L^{k}}{L}\right),
$$

although the physical implications must be explored in order to justify such a hypothesis. In any case additional use of the self-similar closure must be made to finally close the equations.

In Chaboyer and Henriksen(1990) the feed-back to the dynamical equation was ignored completely so that equation (17) was not used. The magnetic field behaviour is however assumed to be dictated by the velocity field in that the self-similarity symmetry of equation (16) is fixed by using the same global constants that appear in the dynamical treatment of non-magnetic turbulence (Henriksen,1988). This essentially supposes equipartition magnetic fields in the ensemble average, since otherwise there would be no reason for such self-consistency.

The details of the solution do not concern us here, but it is worth reporting that the solution for $U$ (neglecting the divergence term) that is consistent with the scaling laws discussed in section 1 for $D_{\rho}=1$ is

$$
U=\text { const }+\frac{t^{2}}{L}
$$

This indicates that at large separations the 2-point magnetic correlation tends to a constant, and that it grows in time at a fixed separation presumably as a result of stochastic diffusion from the small or protostellar scale where the simple theory has it diverging with the density. In fact we deduce that

$$
B_{r m s} \propto \sqrt{\rho},
$$

on the small scales. This behaviour indicates an outward diffusion of magnetic flux consistent with the outward diffusion of angular momentum, and of heat predicted dynamically in the turbulent theory, and necessary for the formation of stars. However the effect found here changes the magnetic Jean's mass only $\propto \rho^{-1 / 2}$ which is too slow if the magnetic field is in equipartition on the large scales. This contrasts with the rate of reduction of the Jean's mass and of the 'centrifugal mass' which both vary as $\rho^{-2}$ in the theory. Thus we must conclude that if the field is indeed in equipartition on the large scales then rather dramatic processes of magnetic dissipation must occur on the protostellar scale. However the conclusion is very sensitive to the magnitude of the mean field in molecular clouds (the magnetic Jean's mass $\propto B^{3}$ ).

\section{Acknowledgements}

The author wishes to express his thanks to the Service d'Astrophysique du C.E.A. Saclay for their hospitality and support while this work was completed. 


\section{References}

Argoul,F.,Arneodo, A.,Elezgaray,J.,Grasseau,G. and Murenzi,R. : 1989 , "Wavelet Transform of Fractal Aggregates." Physics Letters A, 135,327-336.

Chaboyer,B. and Henriksen,R.N. : 1990, "Turbulent Magnetic Fields I" Astron. Astrophys.,in press.

Chandrasekhar, S. : 1955a, "Homogeneous Turbulence I" Proc. Roy. Soc. A,233,322.

Chandrasekhar,S. : 1955b, "Homogeneous Turbulence II" Proc. Roy. Soc. A, 233,330.

Chandrasekhar,S. : 1957, "Homogeneous Turbulence III" Annals of Physics,2, 615.

Elmegreen, B.G. : 1989,"A Pressure and Metallicity Dependence for Molecular Cloud Correlations and the Calibration of Mass." Astrophys. Journal,338,178.

Gill A. and Henriksen,R.N. : 1990,"A First Use of Wavelet Analysis for Molecular Clouds" Astrophys. Journal, submitted.

Henriksen, R.N. and Turner,B.E. : 1984, "Star Cloud Turbulence" Astrophys. Journal,287,200207.

Henriksen,R.N. : 1986, "Star Formation in Giant Molecular Clouds." Astrophys. Journal,310,189.

Henriksen,R.N. : 1988, "Inhomogeneous Turbulence" Astrophys. Journal,331,359-369.

Henriksen,R.N. : 1990, "On Molecular Cloud Scaling Laws and Star Formation" Astrophys. Journal, submitted.

Hoyng, P. : 1987a,"On Magnetic Dynamos I" Astron. Astrophys. , 171,348.

Hoyng, P. : 1987b, "On Magnetic Dynamos II" Astron. Astrophys. ,171,357.

Hoyng, P. : 1988, "On Magnetic Dynamos III" Astrophys. Journal, 332,857.

Lattanzio, J.C. and Henriksen, R.N. : 1988, "Collisions Between Rotating Interstellar Clouds" Mon. Not. R. astr. Soc., 232, 565-614.

Moriarty-Schieven, G.H. and Snell, R. : 1988 "The Star Forming Region L1551" Astrophys. Journal,332, 364.

Zinnecker, H. : 1984 "Star Formation from Hierarchical Cloud Fragmentation : A Statistical Theory of the log-normal IMF." Mon. Not. Roy. astr. Soc.,210,43-56.

Zinnecker, H. : 1989 "Star Formation in Galaxies" in Evolutionary Phenomena in Galaxies, (J. Beckman,Ed.), 115, Cambridge University Press. 